\title{
A Comparison of Wrist and Hip Accelerometer Output at Different Walking Speeds
}

\author{
Albert R. Mendoza ${ }^{1}$, Amanda M. Hickey ${ }^{1}$, Allison H. Gruber ${ }^{1}$, \\ John Staudenmayer ${ }^{1}$, Patty S. Freedson ${ }^{1}$, FACSM \\ University of Massachusetts, Amherst, MA $^{1}$
}

Physical activity has been objectively measured using hip-worn accelerometers for decades. However, wrist-worn accelerometers are currently used in large-scale studies. Differences in wrist and hip dynamics during locomotion may affect monitor output, which may impact how prediction models are built.

PURPOSE: To compare ActiGraph ${ }^{\mathrm{TM}}$ wrist and hip accelerations (g's) at varying locomotion speeds. METHODS: Participants $(\mathrm{N}=7)$ wore ActiGraph ${ }^{\mathrm{TM}} \mathrm{GT} 3 \mathrm{X}+$ accelerometers on the dominant wrist and hip (sampling rate $80 \mathrm{~Hz}$ ). They performed three 5-minute trials at self-paced (SP), slow (SL), and fast (F) over-ground walking speeds. Mean and standard deviation of the vector magnitude (VM) were calculated from two 20-s data windows per condition. Linear mixed-effects models were used to determine if the relationship was different between speed and vector VM at the hip and wrist. RESULTS: Significant differences were found between the slopes (speed vS VM) of the hip $m=0.052(95 \% \mathrm{Cl}: 0.033,0.103)$ compared to the wrist $m=0.195(95 \% \mathrm{Cl}$ : $0.160,0.230) p<0.001$.

\section{Location: Hip}

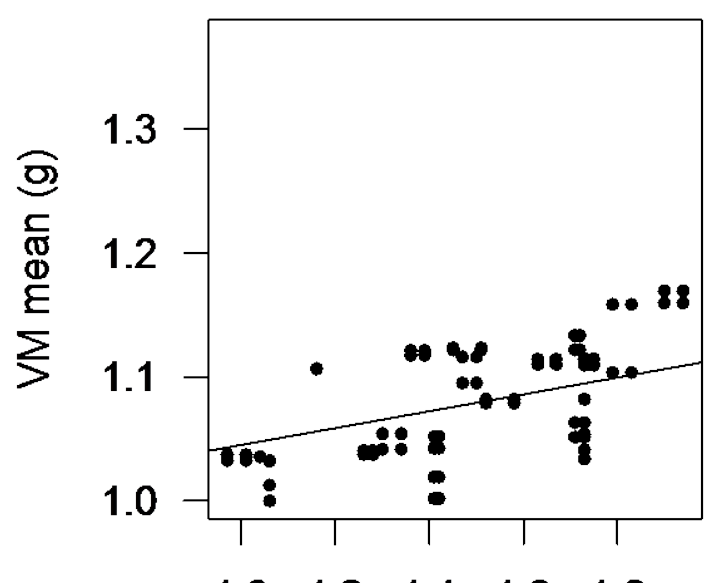

$\begin{array}{lllll}1.0 & 1.2 & 1.4 & 1.6 & 1.8\end{array}$
Location: Wrist

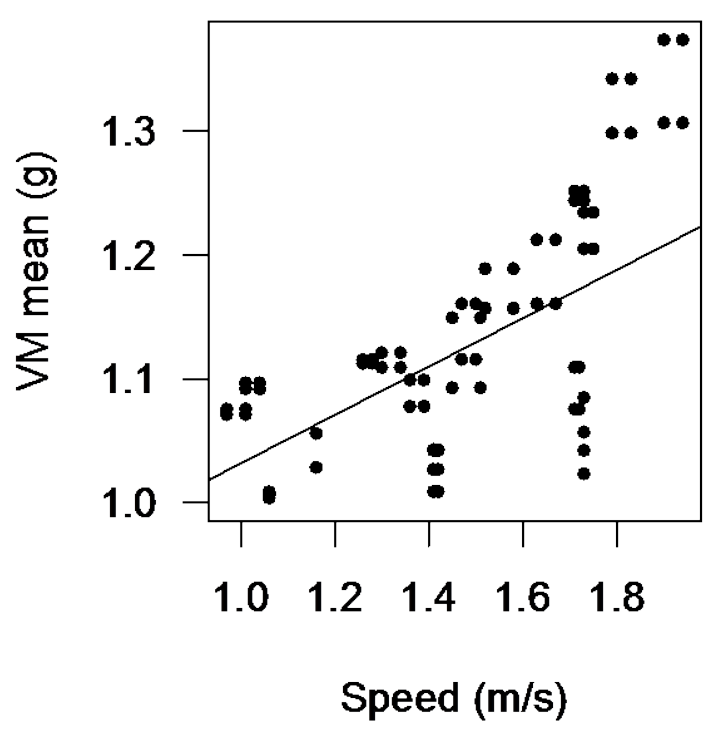

DISCUSSION: The results show that ActiGraph ${ }^{\mathrm{TM}}$ wrist and hip accelerations (g's) differ at varying locomotion speeds. There is a curvilinear increase in VM at the wrist as locomotion speed increases, whereas there is a linear increase in VM at the hip as locomotion speed increases. The pattern of change of wrist VM is different and more variable between subjects compared to hip VM, which may impact measurement error and model development. Additionally, wrist VM is more responsive to changes in speed than hip VM, suggesting that a wrist worn accelerometer may be more sensitive to locomotion intensity. 
Address for correspondence: Albert Mendoza, Department of Kinesiology, University of Massachusetts Amherst, 162 Totman 30 Eastman Lane, Amherst, MA 01003. Email: amendoza@kin.umass.edu 